Vol. 1, No. 1, 2016

\title{
SMART MEASURING INSTRUMENTS FOR CYBER-PHYSICAL SYSTEMS
}

\author{
Mykola Mykyychuk, Svyatoslav Yatsyshyn, Bohdan Stadnyk, Yaroslav Lutsyk
}

\author{
Lviv Polytechnic National University, 12, Bandera Str., Lviv, 79013, Ukraine \\ Author e-mail: slav.yat@gmail.com
}

Submitted on 09.03.2016

(C) Mykyychuk M., Yatsyshyn S., Stadnyk B., Lutsyk Ya., 2016

\begin{abstract}
Aiming the substantial development of CyberPhysical systems, which are becoming a key element of everyday life, the smart measuring instruments are considered below as the indispensable part of entire systems. Verification of the metrological subsystems for parameters determining the controlled equipment and processes through the development, implementation and realization of specific metrology and standardization methods, instruments, that is successfully described by the terms "metrological hardware, software, and middleware".
\end{abstract}

Key words: smart measuring instruments, smart sensors grids, distributed sensors networks, metrological software and middleware, verification.

\section{INTRODUCTION}

Smart measuring instruments (further - MIs) are the prerequisite for CPS design as they constitute the essential units of information and measurement subsystems. There is a set of smart MIs which is divided into the following subsets: smart sensors, smart transducers, their grids and etc. that can be joined together in modern wireless sensors networks (WSNs). Emerging field of cheap and easily deployed sensors offers an unprecedented opportunity for a wide spectrum of various applications. When combined, they offer numerous advantages over traditional networks. These include a large-scale flexible architecture, highresolution data, and application-adaptive mechanisms as well as a row of metrologically specific features and performance (self-check, self-validation, self-verification, self-calibration, ..., self-adjustment).

For instance, special attention according to opinion of Carnegi Mellon University scientists can be given to verification the WSNs in full compliance with wellknown methodology. Timing verification has to guarantee the execution of assigned tasks in real-time WSNs within planned duration. In this area our efforts are directed in developing the schedule-ability techniques for multi-core platforms, where new challenges of shared resources, such as memory, different and changeable algorithms of signal processing and storing, transmission and aggregation have to be considered. Often it would be needed for decision- making in real-time systems, especially in health care and life safety.

\section{SMART SENSORS \& TRANSDUCERS AND THEIR GRIDS}

Main milestones in everyday work aiming the insurance of reliable WSN reliable operation lie in the direction of functional and probabilistic verifications. We provide the software and middleware development aiming to reach predetermined behaviour. The easiest way to achieve this may be demonstrated on the example of widespread wireless fire detectors networks. They are characterized by a number of special algorithms directed on as fast as possible and accurate triggering and actuating the automation of higher level. So, it becomes the necessity of researching and implementing the original operation algorithms for fire sensors and also the checking algorithms for periodic real-time software examination. Considering their structural complexity (presence of smoke and heat sensitive elements, various principles of elaboration of the received signals, their drift of characteristics, and pollution of translucent elements, etc.) development of such algorithms is a daunting task. Herein, human life may be the price of bug. Equally important seems to be probabilistic verification that is assigned to boost the probability of reaching WSN declared goals (estimation of their chances being achieved). This can be fulfilled in two known ways: a) probabilistic model based on Markov approach, b) the similar one based on Monte Carlo simulation techniques.

Significant attention should be given to collaborative autonomy of WSNs designed from the autonomous changeable groups of dissimilar sensors. We try to optimize performance and scalability of them by designing the decentralized operating environment. It may be open- and closed-sourced; the latter is proposed for WSNs that perform mission-oriented tasks. New challenge of CPSs operation is their ability of rapid adaptation to continuous environment alters even unto malfunctions. "Self-adaptive system is the system, capable of changing its behaviour and structure, with 
aim to adapt without human intervention to changes in itself and its operating environment" [1] while considering the WSN's approaches. We obligate to develop not only overall adaptation strategies including predictions of environment evolution but also the specific metrological proactive adaptive mechanisms caused by mentioned changes. If it is the MIs, the arrangements in particular are bound with the change in metrological characteristics and as the consequence with CPS dysfunction. Additionally due to modelling, the adaptations can be performed proactively outpacing the happening drifts and other kinds of performance changes. As result, it spares the time necessary for adaptation strategy that has to be fulfilled.

Smart sensor, according to generally accepted industry definitions, combines a sensing element, analog interface circuit, ADC, and bus interface; all in one housing [2]. Except it, smart sensor includes the microprocessor that conditions the signals before transmission to the control network. Also it filters out undesired noise and compensates for errors before sending the data. Making the grade against the newest generation of smart sensors, however, means that additional functionality must be included, such as selftesting, self-identification, self-validation, or selfadaptation. Of particular interest and importance to designers are such smart sensor capabilities as selfcalibration and self-diagnosis, the ability to use signal processing, and multi-sensing capabilities.

Smart Temperature Sensor is the analog or digital primary thermosensitive transducer combined with a processing unit and a communicating interface [3] and able to perform a row of smart metrological functions due to installed metrological software. This is intelligent temperature sensor with a number of specialized algorithms provided in the design or installation stage, i.e. a sensor with such embedded algorithms that are necessary to provide implementation of following specialized metrology functions. Namely, such functions include, f.i. the ability to realize automatic switching of sub-range of measurement, depending on input signal value; automatic self-validation, self-check, selfdiagnostics and etc.; the introduction of adjustments when the action of impact factor takes place; linearization of metrological characteristics; compensation of cold-junction temperature for thermocouples and so on.

High-mentioned autonomous smart sensors being spatially distributed are frequently combined in network assigned to monitor preset or environmental conditions ( $\mathrm{T}, \mathrm{p}$, etc.) and to cooperatively send received and processed data by network to the end user. Set of such sensors is named WSN if specified sensors are interconnected and distributed in space. Furthermore, the most modern WSNs can operate bi-directionally which means that the control of smart sensors becomes enable as well as their software and middleware updating.

\section{DISTRIBUTED SENSOR NETWORK}

Each WSN consists structurally from a large number (up to $10^{3}$ ) of nodes which are the individual sensors that have ability to radio communicate with one or several neighbouring units. Most common WSN is the fire alarm sensors network each branch of which has up to $2^{6}$ sensors that was caused by limiting the length of microcontroller register [4]. Topology of every WSN may differ: star, cluster tree, mesh, up to advanced multihop mesh WSN. Propagation technique between the hops of network can be routing or flooding. Nowadays, the problem arises to adapt the traditional network topologies to contemporary communicating conditions. Its significant segment is described in [5] and considered below.

Early sensor networks used simple twisted shieldedpair implementations for each sensor. Later, the industry adopted multidrop buses (e.g., Ethernet). Now we can apply web-based networks (e.g., the World Wide Web) implemented on the factory floor. Three classic network topologies (point-to-point, multidrop, and web) are wellknown. In the more reliable first one, each sensor node requires a separate twisted shielded-pair wire connection. In a multidrop network, each sensor node puts its information onto a common medium; this requires careful attention to protocols in hardware and software. Afterwards, few networks have provided frequency-modulated signals on wires to carry multiple sensor readouts on FM channels. Furthermore, early WSNs were simple radio-frequency implementations of this topology. They applied RF modems to convert the RS-232 signal to a radio signal and back again. Due to simple FM coding and interference impact, the reliability of such networking does not satisfied principally. Only when it was selected the particular frequency ranges of network operation the complete wireless local area networks were emerged. Remote data acquisition systems of similar topology are frequently implemented with in-field data concentrators and radio transmitter aiming the hosts, where the signals are demultiplexed into original sensor signals.

Once the industry began the migration to multidrop buses, problems associated with digitization began to emerge [6]. Especially, it occurs with point-to-point systems, where a single clock of host could be used to time stamp when the analog signals from multiple sensors were acquired. With distributed smart sensors required to implement a multidrop network, synchronization of their clocks becomes the critical issue and important design parameter. Somewhat different problems are inherent in a web topology which all nodes are potentially connected to all other nodes. Some of them can be eliminated by applying the repeaters and routers to make virtual connections.

Significant interest is emerged to ad hoc networks. They are multi-hop networks consisting of wireless autonomous hosts, where each host may serve as a router 
to assists traffic from other nodes [7]. Wireless ad hoc networks cover a wide range of network scenarios, including sensor, mobile ad hoc, personal area, and other networks. Research activities in the specific field of WSNs include sensor training, security through smart node cooperation, sensor area coverage with random and deterministic placement, object location, sensor's position determination, energy efficient broadcasting and activity scheduling, routing, connectivity, data dissemination and gathering, path exposure, tree reconfiguration, and topology construction. Network topology involves not only the sensor nodes, but also the base stations and cross-layers for normal operation. Cross-layering becomes quite important for WSNs. So cross-layer can be used to make the optimal modulation to improve the transmission performance, such as data rate, energy efficiency, etc.

In accordance with [7] the ideal wireless sensor is networked and scalable, smart and programmable, capable of fast data acquisition, reliable and accurate over the long term, and requires the minimal maintenance. Selecting the optimum sensors and wireless communications link requires knowledge of the application and problem definition. Battery life, sensors update rates, and sizes are all major design considerations. Examples of low data rate sensors include temperature, humidity kinds. Examples of high data rate sensors are the sensors of strain, acceleration, and vibration. Contemporary examples of extra high data rate sensors are thermal and optical imagers, remote control sensors, and others.

Advance in micro electronics have resulted in the ability of sensors, based on digital electronics, and radio communications to develop the integrated units. Such sensors acquire the ability to communicate with each other with help of wireless data routing protocols. The WSN preferably has to consist of a base-station ("gateway") that can communicate with a few of wireless sensors via radio-waves. Data is accumulated at the sensors node, compressed and transmitted to the main or to the intermediate base station.

We are not considered the architecture of WSNs below as it is traditional (star, mesh, hybrid star - mesh). Similarly it is not the special purpose to study ordinary network standards (IEEE802.11x that is used for LANs at high bandwidth data transfer; IEEE802.15.1 and 2 that are known as Bluetooth that is of lower power and applies for personal area network; IEEE802.15.4 that is specifically designed for the requirements of WSNs, here the worldwide licence-free band $-2-4 \mathrm{GHz}$ - is applied; ZigBee which alliance encompasses the IEEE802.15.4 specification and expands on the network specification and the application interface; and one of most modern standard is IEEE1451.5 that aims the operating of smart sensor working groups in their wireless option).
If a centralised architecture is used in a sensor network and the central node fails, then the entire network will collapse, however the reliability of sensor network can be increased by using distributed control architecture. Distributed control is used in WSNs for the following reasons: sensor nodes are prone to failure; for better collection of data; to provide nodes with backup in case of failure of the central node; resources have to be self organized.

A smart grid sensor is a small, lightweight node that serves as a detection station in a sensor network. Smart grid sensors enable the remote monitoring of equipment such as transformers and power lines and the demandside management of resources on energy smart grid [8]. Smart grid sensors can be used to monitor weather conditions and power line temperature, which can then be used to calculate the line's carrying capacity. This process is called dynamic line rating and it enables power companies to increase the power flow of existing transmission lines. Smart grid sensors can also be used within homes and businesses to increase energy efficiency [9]. According to NanoMarkets, companies like GE, LG and Whirlpool have already announced their commitment to building home appliances that are smart-enabled. Smart grid sensors will link these appliances with smart meters, providing visibility into real-time power consumption. Power companies can use this information to develop real-time pricing and consumers can use the information to lower their power consumption at peak times [10].

\section{MIDDLEWARE AND WIRELESS SENSOR NETWORKS}

Middleware layer is a novel approach to fully meeting the design and implementation challenges of WSN technology. We are considering the WSN middleware as a software infrastructure that glues together the network hardware, operating systems, network stacks, and applications. A complete middleware solution should contain a runtime environment that supports and coordinates multiple applications, and standardized system services such as data aggregation, control and management policies adapting to target applications, and mechanisms to achieve adaptive and efficient system resources use to prolong the sensor network's life. Middleware should provide low-level programming models to meet the major challenge of bridging the gap between hardware technology's raw potential and the necessary broad activities such as reconfiguration, execution, and communication.

The relevant middleware projects for WSNs were studied in [11]. In cases where physical contact for replacement or maintenance is impossible, wireless media is the only way for remote accessibility. Hence, middleware should provide mechanisms for efficient processor and memory use while enabling lower-power communication. Major WSN properties are studied 
below. Scalability is the first one and is defined as follows: if an application grows, the WSN has to be flexible enough to permit this growth anywhere and anytime without affecting network performance. Efficient middleware services must be capable of maintaining acceptable performance levels as the network change its dimensions and topology. The latter is subject to frequent changes owing to factors such as malfunctioning, device failure, mobility, and so on.

Most WSNs' applications are real-time phenomena; so, middleware should provide real-time services to adapt to the changes and provide consistent data. Application knowledge's design principles dictate another important and unique property of WSN middleware. Middleware must include mechanisms for injecting application knowledge of WSN's infrastructure. This lets developers map application communication requirements to network parameters, which enable them to fine-tune network monitoring. WSNs are being widely deployed in domains that involve sensors information for example, in healthcare and rescue areas. The untethered and large deployment of WSNs in harsh environments increases their exposure to malicious intrusions and attacks such as denial of service. In addition, the wireless medium facilitates eavesdropping and adversarial packet injection to compromise the network's functioning. All these factors make security extremely important. Furthermore, sensor nodes have limited power and processing resources, so standard security mechanisms, which are heavy in weight and resource consumption, are unsuitable. These challenges increase the need to develop comprehensive and secure solutions that achieve wider protection, while maintaining desirable network performance. Middleware efforts should concentrate on developing and integrating security in the initial phases of software design, hence achieving different security requirements such as confidentiality, authentication, integrity, freshness, and availability [12-13].

\section{SPECIFICS OF MEASURING INSTRUMENTS DUE TOTHEIR SMARTNESS}

Analog interface is a set of MIs that are an integral part of measuring channel between the primary measuring transducer and ADC. It performs the following functions: scale transformation of measurement signals, their filtering, temperature compensation of thermocouples cold junctions, galvanic separation of transferring and receiving parts, linearization of characteristics and initialization of passive MTs, measuring signals multiplexing, measurement and service information transmission.

Smart sensors are supplied with digital information transmissive means by equipping them with built-in digital controllers to match the universal network interface or by combining technology of analog and digital transmission in a single measuring channel. By the structure all smart sensors are divided into 4 groups: sensors of centralized and decentralized types, as well as sensors with digital and analog buses. By correction methods the analog interfaces with smart sensors are divided into the groups: with manual error correction, with auto correction of errors in analog-digital form, and with digital correction of errors.

It is imperative the further development of $\mathrm{R} \& \mathrm{D}$ works regarding the assessment of accuracy in field of metrology in particular while single measuring the multivariable values by using multiparameter smart MIs, for instance Coriolis mass flowmeters (CMF) [14]. They can be considered to flexible tools and equipment to be able to quickly re-engineer existing procedures. Resultantly, by choosing the right measurement technology solution we can efficiently supply both development and production with relevant data.

CMF transformation function is the dependence of the liquid mass or volume on its flowing velocity through the specified cut. It is determined by the comparison of the time characteristics of two identical sensors in the CMF input and output. More phase difference of mentioned characteristics corresponds to faster controlled environment flowing. So there is a dependence on the hydrodynamic regime of the current environment flowing through the CMF, its viscosity, the temperature etc. Within the current metrological conception the CMF consists of transducers with appropriate sensors and peripheral devices and the microprocessor unit of received signals processing. The CMF sensors determines the flow velocity, temperature and provide information in form of output signals to the microprocessor that carries out the function of the brain of the measurement device and system in total providing access to the display, main menu and output device of processed information for the interaction with other systems, for instance, the filling system. Peripheral devices provide monitoring, warning signalization and other functions, for instance, periodic processes management and the function of liquid density more accurate determination etc. The CMF transfer function error temperature component is the error specified by the temperature regime of liquid/gas flowing. It depends on the temperature of the control environment (on temperature dependence of the liquid flowing regime through the $\mathrm{CMF}$ ); on the $\mathrm{CMF}$ body outlet temperature that is provided with the help of temperature detecting means for detecting a temperature of the inner tube, and temperature detecting means for detecting a temperature of the outer tube; temperature correcting means for compensating an instrumental error according to a change of temperature of the inner tube; temperature difference correcting means for compensating an instrumental error according to a difference between a temperature of the inner tube and a temperature of the outer tube.

Next example of the necessity to develop smart MIs could be industrial tomography systems. Their 
development consist in development and implementation of methods and algorithms to process the results of measurements which would enable for a limited amount of gauges exactly and quickly obtain high-quality images of the distribution of the studied variables. Mainly it would be the spatial distribution of the electrical conductivity of the medium. Improvement of the exactness and rate of tomography measurement have been reached recently [15] by reducing additive gauge errors through the use of the difference measurement method; by elaborating the particular methodology for calculating the sensitivity matrix without of methodical errors inherent in finite-difference methods and based on only a unitary solution of the direct task; ensure the stability and convergence of the iterative procedure of the spatial distribution determination.

\section{VERIFICATION OF METROLOGICAL SOFTWARE AND MIDDLEWARE}

Presented here approach substantially differs from generally accepted one. The latter is described in the following subsection. Commonly as the software metric is considered a standard of measure of a degree to which a software system or process possesses some features. Since quantitative measurements are essential in all areas, the goal is obtaining objective, reproducible and quantifiable measurements, which may have numerous applications in schedule and budget planning, cost estimation, quality assurance testing, software debugging, software performance optimization, and so on.

Our specific measurement consists in evaluation of MIs performance reliability, trueness, and other metrological properties, due to the quality of the certain kind of metrological software, or the software linked with metrological features of MIs.

Design and development of MIs involves the reduction of analog units and expansion of digital ones due to the advances of last. These include the digital microcontrollers, programmable logic arrays and more. Trend of modernity consists in growing the "weight" of software in measurement means. Inconsistency of software to the measuring tasks of certain MI, accidental or intentional alteration of its functions can lead to incorrect measurement results. It is therefore advisable to conduct verification of software to derive its impact on the metrological characteristics of MI and the possibility of further running the studied software as a consisting part of the device, tools, or metrological mean [16].

Under the MI's software we mean a set of programs and procedures designed to register, aggregate, process, display and save, and post the measurement results. Such software as a functional part of MI is delivered jointly with hardware [17-18]. According to [18] software, that may affect metrological characteristics of MIs, concerns: a) programs and program modules that participate in processing of measurement results; b) involved in the calculations the software parameters that affect the results; c) programs and program modules that carry out presentation of measurement data, its storage and transmission, software update and identification, secure software development [19] and data protection; d) components of protected interface for data exchange between software modules of CPS units.

The last 2 items are appropriately attributed to overall not metrological verification of software, since no measuring or computing actions that affect the measurement results, have been fulfilled. These points characterize the correctness of the software functioning in general. So, we have the expertise to incorporate security practices - authentication, authorization and auditing into each phase of the verification, from software design and implementation to testing and deployment [20]. Secure software engineering has become an increasingly important part of software quality, particularly due to the development of the Internet. While IT security measures can offer basic protection for the main areas of our IT systems, secure software is also critical for establishing a completely secure CPS's environment. Every single software MI developer must care about security, because users need to be able to trust the proposed software including the MI software and middleware. Entire cover cycle of the aforementioned wares development includes requirements engineering, trust \& threat modelling, secure coding, security testing, and security response to code protection [21].

MI software metrological verification raises the problem of choice the appropriate methods of software and middleware assessing, testing, and certifying. Result of the metrological validation must be the confirmation or negation of studied ware to the requirements noted in normative documents. Procedures and methods of checking software, and determining its disadvantages are considered below. Software study include first of all the fulfilment the procedures of inambiguity ensuring the operating functions for generated data. Selection of the procedures is defined by regulation requirements, as well as by software developer or user desires to confirm its compliance with target specification.

To validate the type of MI, test procedure should envisage an identification and evaluation of software impact on metrological characteristics as well as to prevent unauthorized software reconfiguration and interference that can degrade the trueness of received measurement results. Therefore the developed under tests, project description of MI type should contain additionally to metrological characteristics the description of software, identity, impact assessment, and the level of protection against unintentional and intentional changes. Certification of MI software and middleware is the research that aims the determination of characteristics, features, identification data, and confirmation of requirements compliance. In accordance with methodology of software certification to determine one or more characteristics (analysis of documentation and source code, functional inspection under controlled conditions, etc.) the tests are carried out [22]. 
Distinguish two kinds of certification: general and metrological. The object of general appraisal is the complete software, study of which is conducted to justify the application of algorithm (program) within specific tasks. To assess the impact of software on the data inexactness, the metrological certification examines merely that software which is an integral part of complete software of the particular MIs. Software certification is mostly voluntary except the software that performs especially responsible functions, where the lack of quality, mistakes or failures can severely disrupted or are dangerous for life and health (aviation, nuclear power, management of authorities and banking systems, etc.) [23].

It is important to choose the correct verification method for MI software and middleware. There are considered the possibility and cost of its implementation, the checking quality and more.

Method of comparative testing with applying the reference software is used for the certain software which helps to identify its features being checked. As the reference software can be studied: attested and/or certified software, functionality of which is similar to tested software features; specially developed software with functions that are identical to functions of software that has to be checked; software for assignments of computing tasks (e.g. spreadsheets, software for mathematical and statistical calculations, etc.).

To development of reference software, somebody may resort in cases when the software under test is not too complicated and its implementation algorithm is rather simple. Reference software should not reproduce all the functionality of software that is tested and could only contain functions and parameters that affect MI metrological characteristics. In certain cases, the peculiarities of graphic user interface and functions that do not participate in the handling of measurements (e.g., display functions, data storage, etc.) are not taken into account. Given method enables to consider the metrological characteristics and maximum peculiarities of software that is tested. Drawback of this method is that often the complexity of software implementation makes it inexpedient due to high costs of developing the reference software.

In absence of reference software, the priority is given to the method of comparative trials by using output data models and comparative tests with the generating the "standard" data. The latter is recommended for certification of data processing algorithms of measurement results. Method enables to evaluate the algorithm possibilities by comparing of processed results of output data model obtained with help of mentioned algorithm, towards the specified parameters of this model. The method of output data models is a kind of generation method of "standard" data, not just data generated by specially developed programs; only data are not generated by the specially developed program, and programmatically they are set at the input of software which is tested. Output data models are selected in a way that they fully regard the measuring tasks covering the greatest range of possible values. These may include: data that completely cover the range of possible values; data close to the largest and smallest values as well as several intermediate values; specific values of input variables - points of the sharp rise or rupture of derivatives, zero, single, and extremely small numerical values of variables, etc.

If values of a variable depend on values of another variable, the test is carried out for the certain combinations of these variables, such as the equality of two variables, or large and small their difference, or zero values of variables. Test method of output data models is easier to implement than the classic method of generating "standard" data. However, development of this method requires a priori information about software algorithms and their program realization, which it is not always known.

Method of generating "standard" data, as the method of output data models, is applied alternatively to the reference software method, or in the case of inability to check the particular functions of implemented software. One of the prerequisites of method of generating "standard" data is the availability of a priori information about the corresponding measuring task. "Reference" data is produced by specially developed generating program. i.e. the generator of "standard" data based on specified output data. The last realize in one of programming languages or by using the standard mathematical (statistical) software package. Initial data for testing, including for the generation of "reference" data, are formed considering the properties of software algorithms that are checked. Method of generating "standard" data is an alternative one to the method of using the reference software. On the other hand, development generator of "standard" data is advisable as it is cheaper than other methods of implementation [24].

If there are several program realizations of the same measurement algorithm at absence of the reference software, is expedient to carry out checks by comparing method implementation. According to it, the same set of "standard" data is submitted to inputs software products, and comparison of relevant test results is performed. Comparison method is simple and requires no the extra programs. However, programs with the same functions are quite rare.

When testing on an analysis of source code of software, the following items are checked: conformity of algorithms structure to the rendered documentation; correctness of recorded algorithms to the chosen programming language; matching the selected algorithms up measuring tasks (detection of unstable algorithms).

When checking the compliance of algorithms structure to the rendered documentation, the algorithms block diagram can be composed and compared with the algorithms described in documentation. In the event of differences between the structures of algorithms, the 
additional analysis elements block designs is conducted. When checking the correctness of algorithms to the chosen programming language, the compliance of code to programming regulations, the presence of uncertain variables and operators, the correct organization of cycles and so on are established. Conformity of selected algorithms with measuring task can be estimated by mathematical analysis of implemented software algorithms. It can be explored algorithms of realized different characteristics, in particular, may be executed an optimal analysis of numerical methods for solving measurement tasks. This method provides an opportunity for detailed assessment of metrological software. However to implement test method on analysis of source code, the experts in software industry and in metrology must be engaged simultaneously. So, costs for implementation of it are much higher compared to other methods.

Middleware, being downloaded from the Internet and installed in MIs, is the software that provides services to software applications beyond those available from the operating system of programmable block of MI. It is often can be presented as "software glue". Middleware makes it easier for software developers to fulfil communication and input/output, so they can concentrate the attention on their own problems. Middleware is the software layer that lies between the operating system and such an application as WSNs. As a result, it supports complex, distributed business software applications.

Middleware includes Web servers, application servers, content management systems, and similar tools that support application development and delivery. It is especially integral to information technology based on Extensible Mark-up Language (XML), Simple Object Access Protocol (SOAP), Web services, SOA, Web 2.0 infrastructure, and Lightweight Directory Access Protocol (LDAP), which is commonly used for communicating and managing in distributed applications.

In simulation technology, middleware is generally applied in the context of high level architecture to a number of distributed simulations. This is a layer of software that lies between the application code and the run-time infrastructure. Middleware mainly consists of a library of functions, and enables a row of applications simulations or federates in HLA terminology - to page these functions from the common library rather than recreate them for each application. Wireless networking developers can use middleware to meet the challenges associated with WSNs. Implementing a middleware application allows developers to integrate operating systems and hardware with the wide variety of various applications that are currently available. For instance, radio-frequency software toolkits provide middleware to filter noisy and redundant raw data.

\section{METROLOGY AND MODELLING FOR ADDITIVE MANUFACTURING}

Increasing demands for precision measurement raises the new problems of optimization of mathematical models of measuring transformations, and adequate processing of experimental data. This problem in modern MIs is particularly relevant due to the capabilities of inexpensive hardware implementation in basis of modern microelectronic components that opens the possibility of computing realization directly in the measuring path.

Obtaining the necessary precision in many cases is only possible in the case of optimal mathematical models that provide in certain sense the best approach of MI general transformation function. Rational choice of mathematical model of transformation function often improves the measuring accuracy or expands the measurement range of preset accuracy.

In this regard, becomes important choice of suitable criterion during processing the experimental data. Normal criteria mostly applicable for analyzing experimental data, is the most common method of mean squared errors, which consists in minimizing the sum of squares of the errors and computing the average of these squares. Unfortunately, the root-mean-square approximation does not provide achievement of the lowest difference between the estimator and function that is estimated at all points of observation, which is desirable during the precision processing of experimental data. Therefore, for solving the calibration tasks should be used the minimax criterion which ensures the minimum possible errors of reproducing the experimental calibration characteristics.

Physical modelling is an experimental method of scientific research, which implies the substitution of the studied physical process by other similar to it of the same physical nature - by model. Physical model is a smaller or larger physical copy of an object. The geometry of model and object that represents it, are often similar in the sense that one is a rescaling of the other; in such cases the scale is an important characteristic. Geometrically similar to the original the model can be both reduced and increased in the comparison with original sizes, and the model of process or phenomenon may differ from the real process by the quantitative physical characteristics such as power, energy, process pressure etc. In a broad sense, any physical experiment conducted in laboratory, including an experiment with natural object or part of it, is a physical modelling. The latter is based on the similarity theory and dimensional analysis, establishing the similarities criteria. The identity of the latter for a nature and the model provides the ability to transfer the experimental results obtained by physiccal modelling, in natural conditions. With the implementation of relevant conditions of physical modelling, i.e. the identity of similarity criteria, the values of variables that characterize a real phenomenon of proportionality of the similar points in space and at similar moments of time, become to be proportional to values of the same variables for the model. Presence of such proportionality allows performing recalculation of experimental results that were obtained on a model by multiplying the value of each of the identified variables 
on a constant for all values a given dimension set factor - the similarity factor [25].

\section{METROLOGICAL PROBLEMS OF ADDITIVE MANUFACTURING}

Advances in AM processes and systems caused by the necessity of design-to-product transformation have resulted in evolution of measurement science, namely in the conjugated material characterization and their performance qualification as well as in process sensing, monitoring and model-based controlling of particular stages of CPS's operation. Since the AM provides "the agility needed to rapidly make innovative customized complex products and replacement parts that are not realizable by more traditional manufacturing technologies or are required to be produced in low volumes" [26], it is especially demanded by CPS-based technology. Therefore NIST Program focuses on overcoming the hurdles, f.i., of 3D-printers widespreading resulted from metrology insufficient development in aforementioned area. Such an optimized system requires the tightly integrated software and hardware components with well-understandable interactions. That is why it should be studied below the contribution of these components into the total efficiency. Unfortunately, 3D-printers, especially printers that perform the units from unique compositions, are currently supplied to market as closed "black boxes".

$\mathrm{We}$, as the process developers, try to access internal hardware and software components with integrated new sensors, models, or control algorithms oblivious to AM vendors [26].

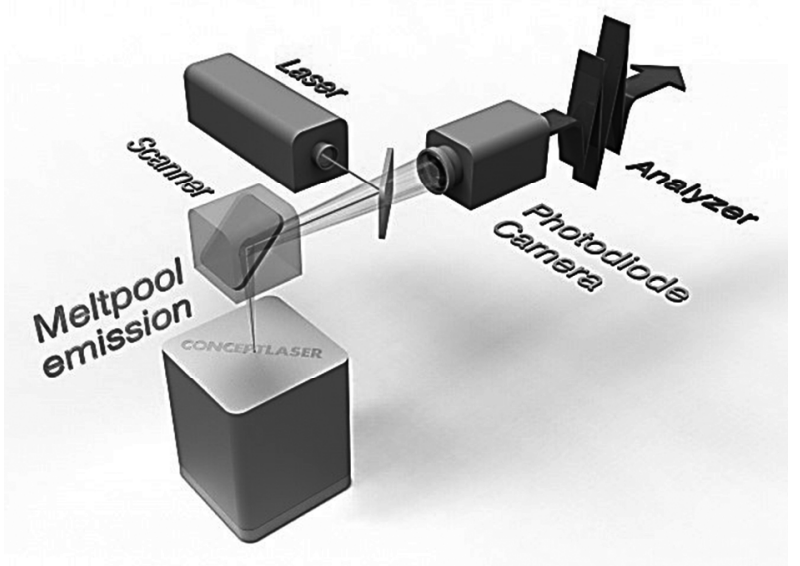

Local monitoring the melt area with help of system QMmeltpool 3D: photodiode and camera are responsible for coaxial monitoring [28]

Researches of Selective Laser Sintering technology have been conducted in this way [27] basing on their own measurements. Here the Quality control is one of the basic requirements. Monitoring the key parameters of laser melting of metallic powders - temperature, oxygen content, power of the laser, and quality of powder - have become a part of modern standards. However, it is impossible to describe exhaustively the product quality on the basis only of equipment parameters. Systems of controlling the local processes in-situ, created by coaxial principle, are desperately needed. In such away it could be received the information about defects that arise during manufacturing.

For monitoring the processes system QMmeltpool 3D [28] in real time provides data that determines the products quality, and records parameters of the melt zone with spatial reference, ensuring the 3D-visualization for further analysis which is similar by HD-resolution to computer tomography. The received result is exceptionally accurate 3D-description of the product, or more truly the definition of characteristic features of deposited layer (measured by photodiode and camera data on area and temperature of the melt zone that are conjugated with relevant data on the laser beam location). General data are visualized in 3 coordinates immediately, gaining the opportunity to track the process of creating each detail in space. This approach simplifies the identification of individual defects in obtained product. Due to the high resolution and frequency of digitization (every $0.1 \mathrm{~mm}$, depending on the speed of scanning), the mentioned system simplify the identification of defects at early manufacturing stages as well as avoid them in the future. Consequently, there arises the possibility to optimize production and technical developments through iterative parameter variation. It can be reached through a two-way process, firstly, by software development on correct description of the details' surface while manufacturing or reconstructing, or, secondly, by further technology development of LayerWise manufacturing of products of given shape from specific, for example, a composite material.

\section{CONCLUSIONS}

Implementation of Cyber-Physical Systems is impossible without easily deployed Smart Measuring Instruments that suppose an opportunity for a wide spectrum of various applications. Numerous advantages include a large-scale flexible architecture, highresolution data, and application-adaptive mechanisms as well as a row of metrologically specific features (selfcheck, self-validation, self-adjustment, etc.) which unprecedentedly improve their performance.

Predictable ways of Smart Measuring Instruments development consist in further studying the Smart Sensors Grids with improved parameters that are reached not only by software \& middleware enhancing and metric introduction, but also by ensuring their scalability, flexibility and a number of other characteristics as well as by providing the specific metrological checks, for instance, of software, or by model-based diagnostics of Smart Instruments reliability.

Particularly clearly the similar challenges for smart metrology are manifested on the example of metrology for AM purposes, which operates on a double 
transformation, especially on building a computer model of created object, and then on product's realization behind this model. In such a way, the method of segmental polynomial approximation based on invariance of signal intensity of white-light interferogram from optical path difference, may be applicable for surface reconstruction at complicated and nonstationary surface topology [29].

\section{REFERENCES}

[1] A. Platzer. Carnegie Mellon University. Logical Foundations of Cyber-Physical Systems. http://symbolaris.com/logic/lfcps.html

[2] C. Mathas, Smart Sensors - Not Only Intelligent, but Adaptable, Contributed By Electronic Products, 2011-09-29.

[3] S. Y. Yurish, Sensors: Smart vs. Intelligent, Sensors \& Transducers, Vol. 114, Issue 3, March 2010, pp. 1-6.

[4] B. Deb, S. Bhatnagar, B. Nath. A Topology Discovery Algorithm for Sensor Networks with Applications to Network Management, CiteSeerX, 2002, http://citeseerx.ist.psu.edu/viewdoc.1886.

[5] Wayne W. Manges, Oak Ridge National Laboratory.

[6] Ad-hoc Sensor Networks http://www.brunel.ac.uk/cedps/ electronic-computer-engineering/research-activities/wncc/ad-hocsensor-networks

[7] Sensor technology handbook, Editor-in-Chief Jon S. Wilson, SA, 2005.

[8] http://searchnetworking.techtarget.com/definition/smart-grid-sensor.

[9] What is smart grid sensor? - Definition from WhatIs.com. internetofthingsagenda.techtarget.com/definitio...

[10] Change into UA. Start-up in energetics.

[11] H. Salem, M. Nader, Middleware: Middleware Challenges and Approaches for Wireless Sensor Networks, IEEE DISTRIBUTED SYSTEMS ONLINE 1541-4922, Published by the IEEE Computer Society, Vol. 7, No. 3; March 2006.

[12] "What is Middleware?". Middleware.org. Defining Technology. 2008. Retrieved 2013-08-11.

[13] S. Hadim, N. Mohamed, 2006). Middleware challenges and approaches for wireless sensor networks. IEEE Distributed Systems Online, vol 7. Issue 3. 2006, Retrieved March 4, 2009 from IEEE Distributed Systems.

[14] M. Kazahaya, A Mathematical Model and Error Analysis of Coriolis Mass Flowmeters, IEEE Transactions on Instrum. and Measurement, Vol. 60, Issue 4, 2011, P. 1163-1174.

[15] D. Spitzer, The Consumer Guide to Coriolis Mass Flowmeters, Seminar. Spitzer and Boyes, LLC, 2004.

[16] The procedure for certification of software of measuring instruments. Access to information:: http://www.uazakon.com/.

[17] WELMEC 7.1, Edition 2 Information document. Development of software requirements. Vienna, 2005, $48 \mathrm{p}$.

[18] MI 3286 - 2010. Testing of software protection, with the determination of its level at the measuring instruments tests in order to type approval, Moscow, 2010,33 pp. (in Russian).

[19] Microsoft Secure Software Development, The 3-rd Conference on minimization of software vulnerabilities in its development, www.microsoft.com/ru-ru/.../mssdcon2013/.

[20] What is CSSLP (certified secure software lifecycle. Searchsecurity.techtarget.com/.../CSSLP-certifie...

[21] ISSECO, the International Secure Software Engineering Council. www.isseco.org/

[22] Обобщенная модель процедуры испытаний измерительного программного обеспечения / Г. В. Злыгостева, С. В. Муравьев // Известия Томского политехн. ун-та. 2011. Т. 318. № 4, С. 62-67.

[23] Метрология, качество и сертификация программного обеспечения / Е. В. Ковалевская. - М., 2004. - 96 с.

[24] O. Oleskiv, I. Kunets. I. Mykytyn, Review of Techniques and Methods of Software Verification of Metrological Means, Lviv, 2014, Publishing House of Lviv Politechnic, No. 75, 2014, http://vlp.com.ua/node/12707.

[25] S. Yatsyshyn, B. Stadnyk, Ya. Lutsyk, L. Buniak. Handbook of Thermometry and Nanothermometry, IFSA Publishing, Spane, 2015.
[26] Measurement Science Roadmap for Metal-Based Additive Manufacturing, May 2013, sponsored by NIST.

[27] S. Ko, H. Pan, C. Grigoropoulos, et al. All-inkjet-printed flexible electronics fabrication on a polymer substrate by low-temperature high-resolution selective laser sintering of metal nanoparticles, IOP Publishing, Nanotechnology, Vol. 18, 2007, 345202, 8 pp., http://dx.doi.org/10.1088/0957-4484/18/34/345202.

[28] Concept Laser Introduces QA Tool for In Process AM: QMmeltpool 3D Available Next Year, July 2, 2015.

[29] B. Stadnyk, E. Manske, A. Khoma, State and prospects of computerized systems monitoring the topology of surfaces, based on white light interferometry, Computational Problems of Electrical Engineering, Vol. 4, No. 1, 2014, pp. 75-80.

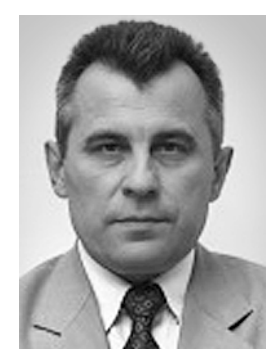

M. Mykyychuk, Dr. Sc., Prof., Academician of Ukrainian Academy of Metrology.

Areas of scientific and engineering research: methods of verification of the measuring tools, solar energetics and its control, means of ensuring the measurement. Author of 2 books and 52 scientific works.

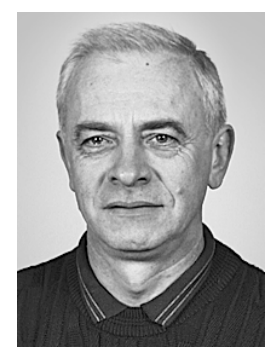

S. Yatsyshyn, Dr. Sc., Prof., Associated Member of International Thermoelectric Academy.

Areas of scientific and engineering research: temperature measurements, nanometrology and nanothermometry, Standards of physical units. Author of 2 books and 156 scientific works.

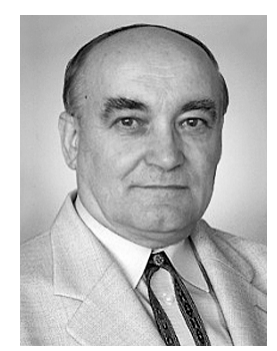

B. Stadnyk, Dr. Sc., Prof., Member of International Thermoelectric Academy, Academician of Ukrainian Academy of Metrology.

Areas of scientific and engineering research: metrology and thermometry, contemporary methods of measurement and standardization, including the intrinsic Standards. Author of 22 books and 473 scientific works.

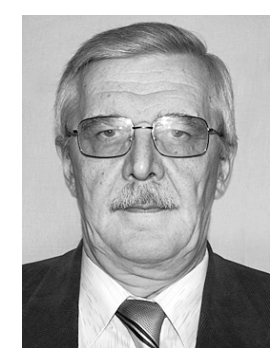

Ya. Lutsyk, Dr. Sc., Prof., Academician of Ukrainian Academy of Metrology.

Areas of scientific and engineering research: acoustic methods of temperature measurement, temperature facilities and instrumentation, nanothermometry. Author of 8 books and 133 scientific works.

\section{ACKNOWLEDGEMENT}

The scientific results, presented in this article, were obtained within the frame of research project number 0115U000446, 01.01.2015-31.12.2017, financially supported by the Ministry of Education and Science of Ukraine. 
